\title{
Current Trends in Modeling Inter-Organizational Cooperation
}

\author{
Authors \\ Antonia Albani and Jan L.G. Dietz \\ Delft University of Technology \\ Information Systems Design \\ Mekelweg 4 \\ 2628 CD Delft \\ The Netherlands \\ \{a.albani | j.I.g.dietz\}@tudelft.nl
}

Abstract.

\begin{tabular}{|l|l|}
\hline Category & General review \\
\hline Purpose of this paper & $\begin{array}{l}\text { Modern enterprises face a strong economical pressure to } \\
\text { increase competitiveness, to operate on a global market, and } \\
\text { to engage in alliances of several kinds. In order to meet the } \\
\text { requirements and challenges of participating in such } \\
\text { alliances, companies must be able to cooperate effectively } \\
\text { and efficiently. This paper provides an overview of some } \\
\text { major directions in inter-organizational cooperation. }\end{array}$ \\
\hline Design/methodology/approach & $\begin{array}{l}\text { In order to cope with the challenges of inter-organizational } \\
\text { cooperation, to share innovative research issues and to } \\
\text { facilitate profound discussions about them, the authors } \\
\text { organized a series of workshops on Modeling Inter- } \\
\text { Organizational Systems (MIOS-CIAO!) starting at the annual } \\
\text { OTM federated conference and continuing at the annual } \\
\text { CAiSE conference. This paper summarizes the results of the } \\
\text { workshops. }\end{array}$ \\
\hline Findings & $\begin{array}{l}\text { This paper provides an overview of what has been } \\
\text { established and what is going on regarding the cooperation } \\
\text { of enterprises in networks. The focus has been on the } \\
\text { modeling of cooperation, from the business level down to the } \\
\text { implementation level. }\end{array}$ \\
\hline Practical implications & $\begin{array}{l}\text { This overview is a useful source of knowledge for those who } \\
\text { want to have a quick insight in the relevant aspects of } \\
\text { cooperation, and in many well known modeling approaches } \\
\text { and techniques. It is also an inspiring source for those who } \\
\text { want to investigate yet unsolved or unsatisfactorily solved } \\
\text { problems. Although developments, both in theory and in } \\
\text { practice, will go on, no landslides are expected. Particularly } \\
\text { for practice, the value of this report will therefore last for a } \\
\text { considerable time. }\end{array}$ \\
\hline What is original/value of paper & $\begin{array}{l}\text { Several core notions in the area of inter-organizational } \\
\text { cooperation are clarified, such as collaboration, cooperation, } \\
\text { enterprise network, choreography, and orchestration. The } \\
\text { whole process of developing or investigating an enterprise } \\
\text { network is covered. }\end{array}$ \\
\hline
\end{tabular}

Keywords. Cooperation, Collaboration, Choreography, Orchestration, Enterprise Network, Business Processes 


\section{Introduction}

The environment in which enterprises are operating today is increasingly determined by intense competition demanding significant changes in the way enterprises do business. In order to compete on the fast changing market, companies are forced to undergo a drastic transformation of business processes as well as organizational and managerial structures (Burtler et al., 1997). The necessity of changing the organization from a function-oriented to a process-oriented structure was already propagated in the nineties, e.g., by (Davenport, 1992, Kaplan and Murdock, 1991, Davenport and Short, 1990, Hammer, 1990), seeing information and communication technology (ICT) as the enabling factor not only to automate but also to redesign the business processes. The concept of business process reengineering and the support of ICT have been widely applied in practice and have led to intra-organizational performance improvements, documented in several case studies as e.g., by (Ross and Vitale, 2000, Scott and Vessey, 2000, Davenport, 1998). Although certainly not all of these projects were successful, the 'BPR movement' set a new direction, both in research and in practice.

In addition, decisive factors such as globalization of sales and sourcing markets, shortened product life cycles, innovative pressure on products, services and processes, and customers' requests for individual products, additionally force companies to identify, improve and (partly) automate their core business processes (Osterloh and Frost, 2003, pp. 28-31, Prahalad and Gary, 1990), while at the same time outsource non-core processes to business partners. This step is crucial in order to better adapt to fast changing market requirements and to become more flexible while meeting individualized customer demands. As a consequence, business processes concerning e.g., product development, market research, sales, production, delivery and services, are affected and have to be adjusted and integrated, not only within a single company but also in a network with external partners. As already recognized e.g., by Malone (Malone and Lautbacher, 1998, pp. 151-152), "the boundaries between enterprises will become much less important. Transactions within organizations will become indistinguishable from transactions between organizations and the business processes, once proprietary, will freely cross organizational boundaries". Companies recognize that the source of their competitive strengths does not only lie in their core competences, but also in the ability to cooperate with their business partners (Jarillo, 1988, p. 31).

To an increasing degree traditional organizational structures evolve towards hybrid and network structures (Picot et al., 2003, p. 289, Malone and Lautbacher, 1998, p. 166) using modern ICT developments - like the Internet, semantic standards, distributed applications, component based applications, and service-oriented architectures - in order to sustain the creation and management of networks (Kopanaki et al., 2000) and to reduce transaction costs (Williamson, 1991). However, due to several project failures in the realization of interorganizational systems - e.g., Nike's incorrect implementation of the supply chain management software of "i2" led to an overproduction of 5 million pairs of shoes (Wilson, 2001) many of those initiatives have stopped. But, despite the existing problems in the interorganizational domain, companies e.g., like Cisco, Dell and Intel, used ICT to implement elements of their business models, enabling to increase market share and to achieve high returns on investment (Anderson et al., 2002, Kraemer et al., 2000). Those business cases provide valuable insights into how ICT can be applied to achieve speed and flexibility in a quickly changing market environment. At present, most of the ICT-enabled networks can be largely found in the form of rather small, flexible alliances of professionalized participants (Etemad et al., 2001).

The support of large networks with multiple tiers of suppliers still causes considerable difficulties. The high degree of complexity resulting from dynamic changes in enterprise networks is the main reason for the lack of practical implementation that is connected with the identification of network entities and the modeling of the network structure, as well as with the high coordination effort, as described in (Lambert and Cooper, 2000). Despite the 
fact that these are basic factors in order to succeed in supply networks, many research efforts have been based on more operative tasks primarily focusing on the optimization of forecast and planning accuracy and the optimization of material flows over the whole supply chain (Houlihan, 1985, Jones and Riley, 1985).

In order to examine the actual state of affairs in respect to the challenges and problems in the area of inter-organizational cooperation mentioned above, researchers met at the MIOSCIAO! workshops and focused on a core set of three questions:

- How to model inter-organizational cooperation?

- How to model, design and execute inter-organizational business processes?

- What are the benefits and restrictions of emerging standards?

The authors of the paper were the organizers of the MIOS workshops. The contributing workshop participants were: Volker Derballa (University of Augsburg, Germany), Birgit Hofreiter (University of Technology Sydney, Australia), Christian Huemer (Vienna University of Technology, Austria), Agnes Koschmider (University of Karlsruhe (TH), Germany), Jan Mendling (Vienna University of Economics and Business Administration, Austria), Nikolaus Müssigmann (University of Applied Sciences, Germany), Paula Ventura Martins (Universidade do Algarve, Portugal), Yiannis Verginadis (National Technical University of Athens, Greece), Barbara Weber (University of Innsbruck, Austria), Andreas Wombacher (École Polytechnique Fédérale de Lausanne, Switzerland), Jelena Zdravkovic (University of Gävle and Royal Institute of Technology, Sweden).

The discussion results are summarized in this paper, giving an overview of some major directions in the area of inter-organizational cooperation. We start in section 2 introducing different forms of inter-organizational cooperation. While in section 3 we focus on the modeling of enterprise networks, section 4 provides an overview of techniques used for conceptual modeling of inter-organizational business processes. Section 5 introduces different languages and the underlying formalisms used for the execution of business processes and presents issues relevant for mapping conceptual inter-organizational process models to such languages. Conclusions can be found in section 6 .

\section{Inter-Organizational Cooperation}

As stated in the introduction, a promising way for enterprises to increase their global competitiveness is to collaborate with external business partners and especially to form cooperative relationships. In this section we will focus on different forms of cooperative relationships. In order to do that we first give our definition of the terms collaboration and cooperation, since no common accepted distinction of the two terms exist so far, and both terms are sometimes used synonymously. Based on the ideas of cooperative and collaborative learning described by (Bruffee, 1995), we define the terms inter-organizational collaboration and cooperation as follows:

Inter-organizational collaboration refers to the relationship between business partners, in which each enterprise learns and profits from its partners in order to better achieve its own goal. Within inter-organizational collaboration, the enterprises remain autonomous and the relationships can be terminated at any time, without affecting the goals of the single enterprises.

Examples of inter-organizational collaboration are industrial congresses, where enterprises meet in order to e.g., exchange experiences, discuss important and hot topics, and/or meet potential partners. They learn and profit one from another and leave again without affecting the goals of the single enterprises.

In inter-organizational cooperation enterprises work together to achieve a common goal. In such relationships, neither party can compete effectively without the continuous contribution of the other partners.

Several forms of inter-organizational cooperation are possible, of which the most relevant one seems to be the enterprise network. 
If two or more companies are involved in inter-organizational cooperation, an enterprise network structure is created. Enterprise networks are formed to better fulfill specific customer requests providing customized products on time in the right quality and for a competitive price. Especially in large manufacturing companies e.g., in the automotive industry, such networks can span over several tiers. Although enterprise networks have been introduced many years ago by Jarillo, Malone and Miles, (Jarillo, 1988, Malone et al., 1987, Thorelli, 1986, Miles and Snow, 1984), there is no single broadly accepted definition of an enterprise network today. Several expressions exist to define different, or sometimes similar, types of enterprise networks. Terms such as strategic networks (Gulati et al., 2000), alliance networks (Gulati, 1998), economic webs (Hagel III, 1996), business webs (Tapscott et al., 2000), value webs (Herman, 2002), virtual networks (Malone and Lautbacher, 1998) or dynamic networks (Pine et al., 1993), can be found in the literature.

As defined by (Gulati et al., 2000, p. 203), "strategic networks potentially provide a firm with access to information, resources, markets, and technologies; with advantages from learning, scale, and scope economies; and allow firms to achieve strategic objectives, such as sharing risks and outsourcing value-chain stages and organizational functions". Gulati uses strategic networks in a quite general manner, assigning several types of networks - which are composed of inter-organizational ties - as e.g., strategic alliances, joint ventures, long-term buyer-supplier partnerships, to this term. More precisely Gulati defines strategic alliances (Gulati, 1998, p. 293) as "voluntary arrangements between firms involving exchange, sharing, or co-development of products, technologies, or services". The authors of economic webs, business webs, value webs, virtual networks and dynamic networks all discuss the same basic idea of supporting enterprises by means of ICT, primarily the Internet, in order to "shed functions in which they are not competitive to service providers and partners that may have far greater expertise, scale or geographical reach" (Herman, 2002, p. 35).

Malone, (Malone and Lautbacher, 1998, pp. 146-148) mainly sees the increasing importance of "ad-hoc structures", where single business units join together into virtual and temporary network-companies in order to produce or sell goods and services, and as soon as the cooperation finishes the temporary network ends to exist. Hermann (Herman, 2002, p. 31) envisions, that "the traditional value chain, which optimized a sequence of functions for one business, is transforming into a global value web, which can optimize the supply, demand, and product design activities for an entire network of partners. (....) Rather than think in terms of a linear value chain, we think of a value web where material, information, and money flow in parallel, taking multiple separate paths through a complex network of suppliers, service providers, distributors, and customers". Pine additionally adds a dynamic aspect to the enterprise networks in order to make mass customization work, saying that "companies must break apart long-lasting, cross-functional teams and relationships and form dynamic networks" (Pine et al., 1993, p. 114). In mass-customization, where e.g., processes, technology and products need to be reconfigured in order to fulfill the individualized demands of customers, the corresponding enterprise networks cannot remain fixed, but need dynamically to be adjusted.

Enterprise networks, especially in the area of manufacturing industry (e.g., in the automotive industry), are gaining more and more importance. E.g., Dodel (Dodel, 2004) cites in his research on the logistic criticality in the automotive industry a logistics manager of DaimlerChrysler AG, who states that "the processes and skills of the direct suppliers are well known. What is missing is the transparency of the complete supply network, which serves behind the direct suppliers. If a supply problem occurs, great efforts have to be taken to identify the cause of the problem". Additionally, in spring 2004 DaimlerChrysler had to call back 1.3 million cars due to problems with the integration of system modules provided by different supply partners. Jürgen Schrempp, CEO of the company, stated during an interview prior to the annual general meeting, "the company underestimated the complexity of networking partners, who deliver complete system modules". 
As seen from these examples, large networks are still causing considerable difficulties due to the high degree of complexity resulting from dynamic changes in enterprise networks, stated also in the introduction. This is connected with the identification of network entities and the modeling of the network structure, as well as with the high coordination effort. We therefore focus in the next section on the modeling of enterprise networks and use supply networks as a specific type of enterprise networks - for illustration. Considering the network definitions introduced above, we use the term supply network in order to define in the manufacturing industry (e.g., in the automotive industry) a network of suppliers spanning over several tiers and communicating among each other using the Internet. The network has a fix part, namely the original equipment manufacturer (OEM), and a dynamic part, the suppliers, allowing flexible extension and modification of the network when additional competencies are needed. The OEM has a higher degree of autonomy, since he is the requestor for specific products and therefore the initiator of the identification of the network partners.

\section{Modeling Enterprise Networks}

(Contributing authors: Derballa, Müssigmann)

In order to identify network entities and to model their relationships, and therefore the structure of enterprise networks, we first need to identify the objects of importance that contribute to the constitution of an enterprise network. The work of (Albani et al., 2005) contains appropriate concepts for the definition of a supply network. Two main possibilities to form enterprise networks can be imagined. First, a network architect or broker selecting the respective network partners can form a network. Second, an automated self-organizing mechanism can be used to identify network partners in order to amend competencies on all levels of the network. Especially in large enterprise networks as found e.g. in the automotive industry, the identification process is a complex procedure (Lambert and Cooper, 2000) and - when done by hand - is very time-consuming. Therefore an example of an automated procedure to identify strategic supply networks based on the self-organizing mechanism is introduced in this paper (see Fig. 2 and correspondent descriptions below).

For each of the modeling approaches several steps (shown in Fig. 1) are necessary in order to identify, evaluate and select potential suppliers according to the overall goal, which drives the creation of such enterprise networks.

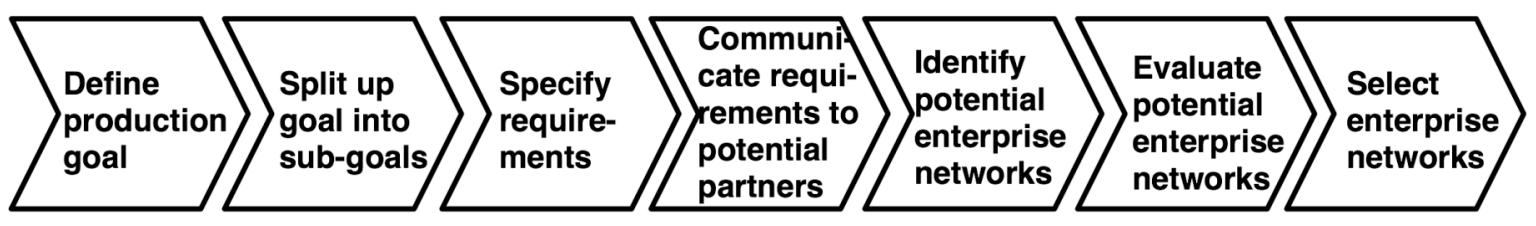

Fig. 1. Process of modeling supply networks

The selection of network partners is following a production goal, which is determined by the benefits for the enterprise and its business needs. The business tasks may be simple ones for instance providing detailed information to a specific product - easily performable by a partner, or more complex ones that necessitate the intervention of multiple partners at different tiers during the lifetime of the enterprise network. If there is no existing enterprise network that satisfies a certain goal, a new enterprise network needs to be created.

To reduce complexity, goals - e.g., manufacturing a car - are split into several sub-goals e.g., producing different parts of the car independently - which are treated separately. Finding partners to accomplish certain sub-goals necessitates that the goals of the partners are compatible with the goal of the initiator. However, goals and sub-goals are still too abstract for selecting partners. Enterprises may need more information about the available capabilities by which the partner goal is to be achieved. Considering the goal 'produce car tires', the enterprise needs to know and constrain many parameters - e.g., the materials to use and its different characteristics - in order to communicate them to potential network partners. This necessitates the definition of a common specification in order to compare the 
different requirements and capabilities. Several standards, as e.g., (eCl@ss, UN/SPSC, n.d.), have been developed in order to have a common understanding about e.g., product types, colors, sizes etc.

Having defined the requested products with a common and standardized specification, the requirements need to be communicated to potential network partners in order to identify potential supply networks. An initiator may find several potential networks of partners that satisfy its need. So the selection of a network (partners) can be done according to evaluation criteria as defined by the initiator. Evaluation criteria may span from simple facts to highly complex considerations. One of the simplest criteria is the minimum number of nodes in the supply network, which can be used to minimize overall complexity of supply networks. Criteria with more complex calculations are e.g., the shortest total delivery time, the minimum total cost or the regional only sourcing. Complex criteria are e.g., maximize product quality or maximize delivery time liability, since these criteria implicate the evaluation of past experience. Algorithms for such an evaluation, as well as their implementation, are described e.g., in (Albani and Müssigmann, 2005). With the selection of the individual network partners the whole network can be modeled. In (Albani et al., 2004b, Müssigmann and Albani, 2006) Albani et al. describe in detail the supply network modeling process and present a corresponding prototype implementation in (Albani et al., 2004a). We will shortly illustrate their identification and selection process. For the identification of potential supply networks, a specific requirement for an offer to a product to be built is specified and communicated from the original equipment manufacturer (OEM) to existing and/or potential suppliers, see Fig. 2.

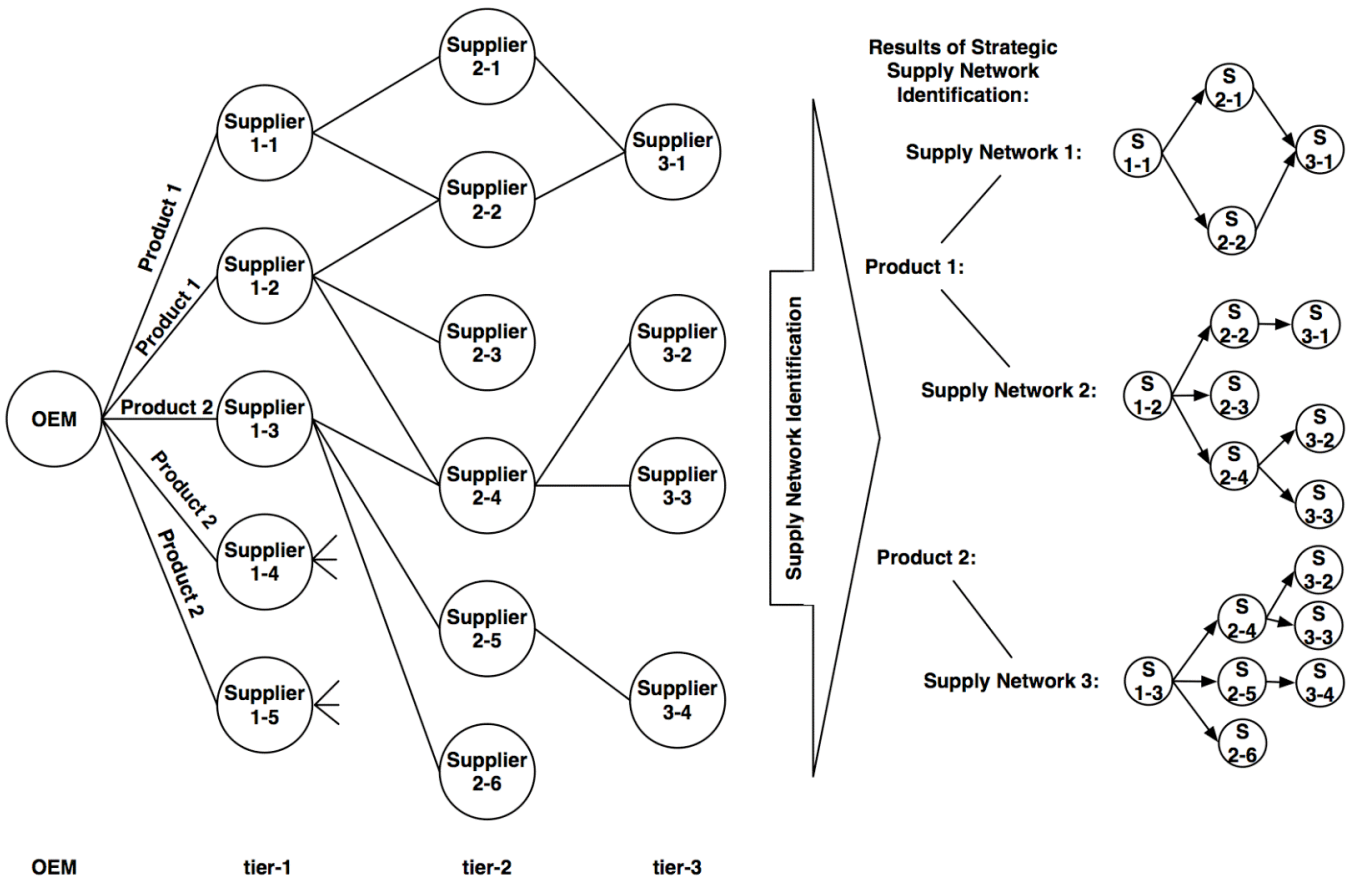

Fig. 2. Modeling enterprise networks (Müssigmann and Albani, 2006)

In the example, the OEM is connected to a potential network of suppliers as shown in the left part of Fig. 2. It is assumed that the OEM needs to order two products externally, product 1 and product 2. During the identification process the OEM sends out demands for these products to its strategic suppliers in tier-1. In the example it is assumed that supplier 1-1 and supplier 1-2 get the demand for product 1 while supplier 1-3, supplier 1-4 and supplier 1-5 receive the demand for product 2 . These suppliers check whether they can fulfill the demand internally and, if not, send out subsequent demands to their respective suppliers. Each node executes the same process as described until the demand has reached the last tier. The requested information is then split-lot transferred to the OEM, aggregated and finally visualized as a supply network, in which each participant of the supply network constitutes a network node. 
This process may result in the identification of several possible supply networks as shown in the right part of Fig. 2, where e.g., product 1 can be provided by two supply networks, supply network 1 (root node S1-1) and supply network 2 (root node S1-2), whereas product 2 can only be provided by supply network 3 . It is now up to the OEM to evaluate and decide which of the potential supply networks (in the example above for product 1) will be selected to fulfill its original demand. The basic information needed for the selection results from the evaluation of potential networks.

\section{Modeling Inter-Organizational Business Processes}

(Contributing authors: Hofreiter, Huemer, Martins, Mendling, Wombacher, Zdravkovic)

With the modeling of the relationships between the network partners, the basis for interorganizational cooperation has been established. In order to perform and compete as an enterprise network, the support of ICT is required allowing to (partly) automate not only the intra-, but also the inter-enterprise business processes. The implementation of such interorganizational information systems requires the interlacing of the business processes of the participating partners. Therefore the business processes of all network partners need to be modeled conceptually, focusing on documenting the flow and the structure of the interorganizational process activities. As stated in the introduction, the goal of business process modeling is to improve existing and/or to design new processes in order to better adapt to fast changing market requirements and to become more flexible, while meeting individualized customer demands.

In order to build a conceptual model of business processes, an adequate modeling technique needs to be selected. The two most widely used techniques for conceptual modeling of business processes are the Unified Modeling Language (UML) (UML, n.d.) and the Business Process Modeling Notation (BPMN) (White, 2004). The main advantage of these techniques is the use of notations that are intuitive to business users, and yet capable to represent most process semantics and interactions. In order to illustrate the two notations, the following inter-organizational example scenario will be used throughout this section:

Example: Three enterprises - a buyer, a seller and a bank - are cooperating and need to interlace their business processes. The buyer sends an initial request for quote to the seller. The seller checks if the requested product is offered, i.e., listed in its product catalogue. If so, then the stock info is required in order to see if the product is kept in stock. If the product is out of stock, product information is needed to check if the product can be produced or not. In cases of either having the product in stock or having to produce the product, the seller needs to calculate its price and to send back a quote to the buyer. If the requested product is not offered by the seller and cannot be produced, a rejection is sent back to the buyer. In case of having received a quote for the requested product, the buyer checks if the price corresponds to the price limit set; if so, it sends a purchase order to the seller. The seller then verifies with the bank the credibility of the buyer. If the credibility is ok, the seller returns an order response to the buyer.

The core elements of BPMN are activities, events, gateways, sequence flows, message flows, pools and transactions. Activity is a generic term for work that a business role can perform. In a BPMN diagram, an activity is represented by a rounded rectangle. An activity can be atomic or compound. A compound activity is composed of other activities and will be marked by a ' + ' sign inside the roundangle. An activity may also be repeated, which is graphically shown by a circular arrow inside the roundangle. Events, represented as circles, are things that 'happen' during the course of a business process. There exist three types of events: start, end and intermediate events. Activities and events are connected by sequence flows, shown as arrows, indicating the order in which activities will be performed or events occur in a business process. Gateways, represented by diamonds, are used to control the sequence flows by determining branching, forking, merging, and joining of paths. Pools are designed in the form of rectangles, enclosing other BPMN elements, separating activities of 
different partners. Message flows, shown as dashed arrows, are used for communication between activities in different pools. The BPMN process diagrams for the example are given in Fig. 3.

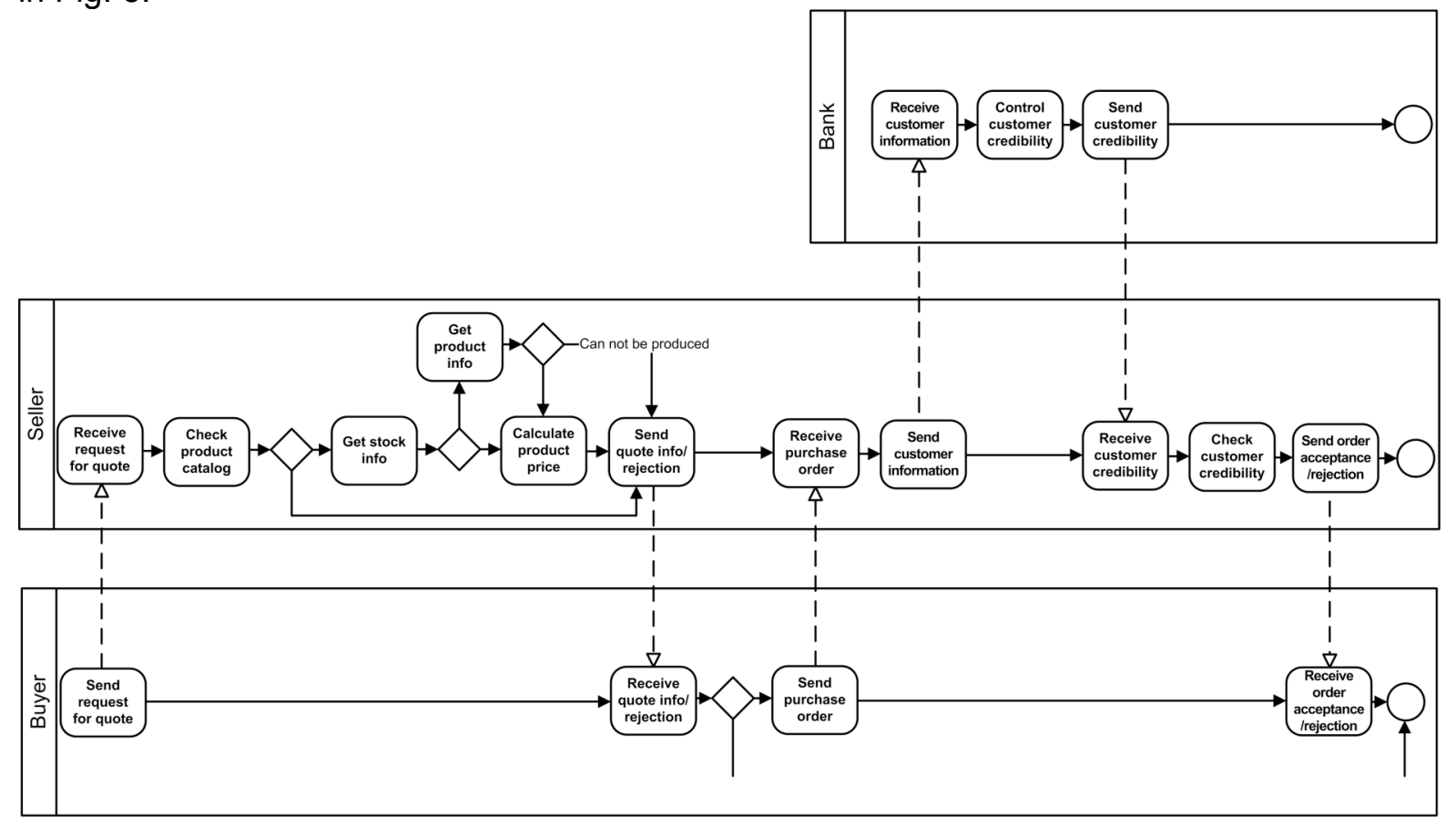

Fig. 3. Inter-organizational cooperation model using BPMN

The Unified Modeling Language (UML) provides graphs, state charts, sequences and collaboration diagrams for modeling the behavior of business processes. There exist a variety of options in UML to model the example scenario by these diagrams. Usually, a software development process or a methodology defines the exact 'how-to' guidelines. Among different methodologies we have selected UN/CEFACT's Modeling Methodology (UMM) (UN/CEFACT, n.d.) that specifies a UML profile dedicated to modeling interorganizational business processes. UMM is used to model interactions between business partners. It does not provide means to describe the activities of business processes that are internal to a partner. During the UMM development process a lot of different artifacts are created. Among those the business collaboration protocol and the business transactions are the most important ones. The term "collaboration" here is a UMM-term; it has nothing to do with the notion of collaboration between companies as discussed in section 2. A business collaboration protocol is an activity graph, which is composed of many business transaction activities. The transitions between these activities are usually guarded by states of business entities that are shared between the business partners. Each business transaction activity is refined by another activity graph called business transaction. Such an activity transaction is composed of two business activities - an initiating activity and a responding activity. A business transaction may contain up to two business documents. The conceptual model of the buyer-seller-bank example, using the UMM/UML notation is given in Fig. 4. 


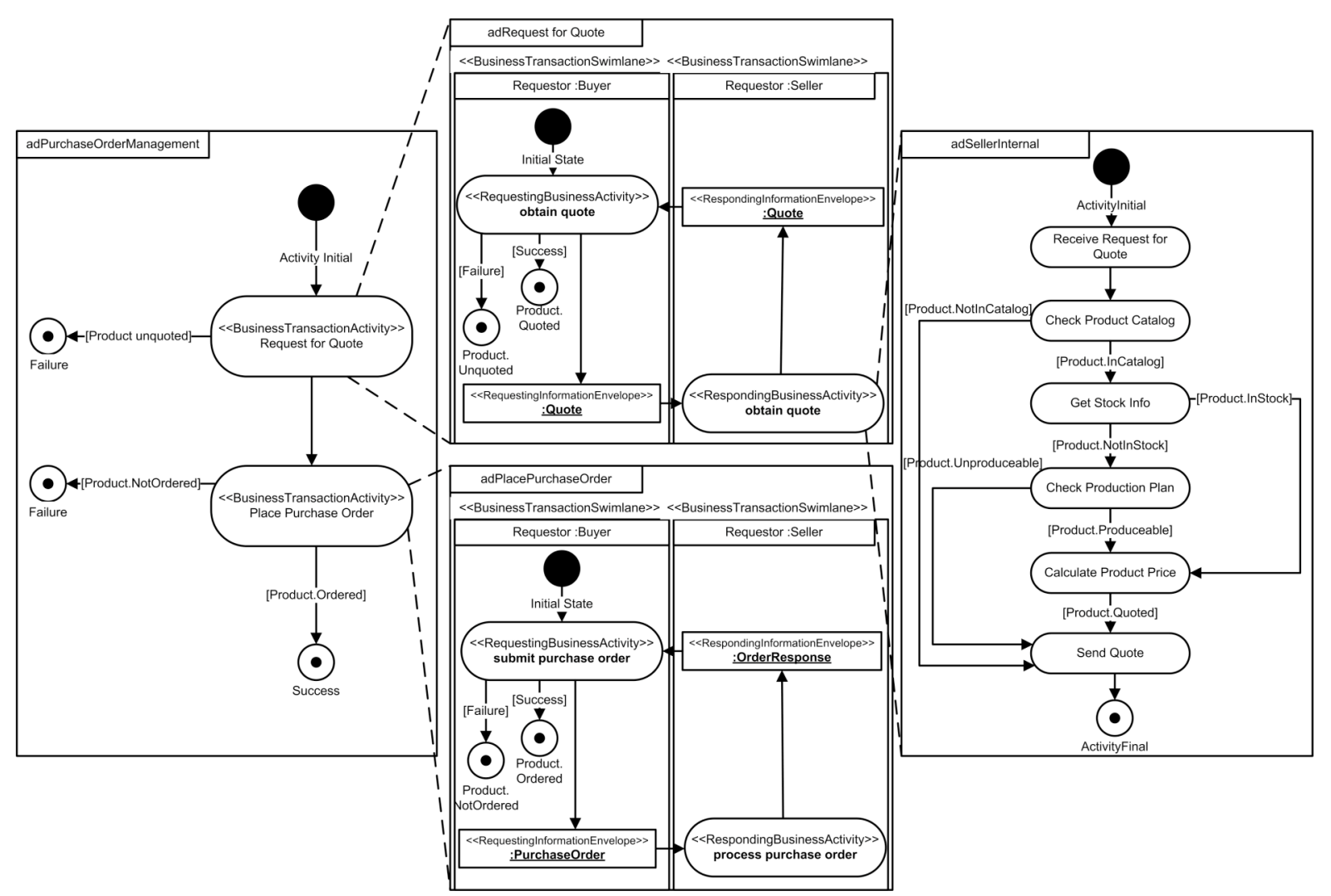

Fig. 4. Inter-organizational cooperation model using UMM/UML notation

The activity graph on the left side of Fig. 4 shows the business collaboration protocol for the collaboration between the buyer and the seller. It consists of two business transaction activities, request for quote and place purchase order, which are refined by the two activity graphs for transactions (shown in the middle column of Fig. 4). It is easy to recognize that the transitions in the business collaboration protocol are guarded by the states of the business entity product, which are set by the business transactions. UMM is used to standardize the global process, concerning interactions between business partners, but is not intended to model the processes internal to an organization. Thus, the activity graph on the right hand side showing the process to calculate a quote is not part of the UMM profile, but still valid UML. Activity graphs, providing a very flexible and loosely coupled concept, may be used to model the internal activities of an enterprise.

Besides the notations used in this section for illustrating the general idea of modeling interorganizational business processes, further approaches exist. However, even if these approaches may use different terminology or notations, the general idea remains the same. For example, Dijkman and Dumas (Dijkman and Dumas, 2004) use a more complete process model considering organizational aspects like roles. Fu et al (Fu et al., 2004) provide an approach based on guarded Finite State Automata, Yi and Kochut (Yi and Kochut, 2004) apply colored Petri Nets, and Muth et al (Muth et al., 1998) use state charts. A very popular notation in business engineering is the Event-driven Process Chain (EPC). It is a business process modeling language focusing on control flow dependencies of activities in a business process (Keller et al., 1992). It is utilized in the ARchitecture of Integrated Information Systems (ARIS) by Scheer (Scheer, 1998, Scheer, 2000, Scheer et al., 2005) as the central method for the conceptual integration of the functional, organizational, data, and output perspective in information systems design. EPCs offer function type, event type, and connector type elements. Functions describe activities of a process and events pre- and post-conditions of functions. A connector has either AND, OR, or XOR logic to define complex routing rules. 
All the different modeling approaches have their pros and cons. The power of a notation in modeling business processes may be evaluated in a comparison of their support of wellknown workflow patterns (van der Aalst et al., 2003). However, it is not the goal of this paper to evaluate and to compare the different modeling languages. The result would most likely be very much the same as for their power to model intra-company processes. Instead we concentrate on which activities have to be modeled when focusing on which abstraction of an inter-organizational system. Further down we use the BPMN, but could have used e.g., UML or EPC as well.

Looking at Fig. 4, two important business process abstraction modeling concepts have been used, namely the distinction between the activities concerning a) only the interactions between business partners and $b$ ) the internal activities of an enterprise. Those concepts are also known as choreography and orchestration and are two new aspects in the context of cooperative business processes. Based on the definition of choreography for Web services given by (Barros et al., 2005) we define choreography in an inter-organizational cooperation context as follows:

Choreography refers to the relationships between business activities, capturing the interactions and their dependencies, in which the participating business partners engage to achieve a common goal. Choreography does not describe enterprise internal business activities that do not directly result in an interaction with another participating partner. This means that choreography encompasses all interactions between the participating enterprises that are relevant with respect to the choreography's goal.

Like for the definition of choreography, our definition of orchestration is based on the definitions of Web service orchestration given by (Alonso et al., 2003, p. 257, Barros et al., 2005, p. 65) and has been adjusted for inter-organizational cooperation as follows:

Orchestration refers to the order and the conditions in which both types of business activities, interactions and internal activities, are invoked. While the interactions are described at message level (e.g., receive and send activities), including the business logic and the execution order of the interactions, internal activities comprise data transformations and invocations of business activities executed e.g., by internal information systems or by human beings.

In order to better explain the definitions just given, we will use the buyer-seller-bank example and model the relevant business activities for the choreography as well as for the orchestration, using BPMN. While modeling the choreography of the example case, all interactions between the enterprises involved in the business scenario need to be considered, resulting in the following activities: The buyer sends a request for quote; the seller receives a request for quote and sends the quote or rejection to the buyer. The buyer receives the quote or rejection and sends a purchase order (or not). The seller receives the purchase order, and sends a credibility request to the bank. The bank receives the customer information and sends customer credibility to the seller. The seller receives the buyer credibility status and sends an order response to the buyer. The buyer receives the order acceptance or rejection of the seller. The BPMN model of the choreography is shown in the top pool of Fig. 5. This type of choreography is also called global choreography, because it includes the single interactions of all participating partners. 


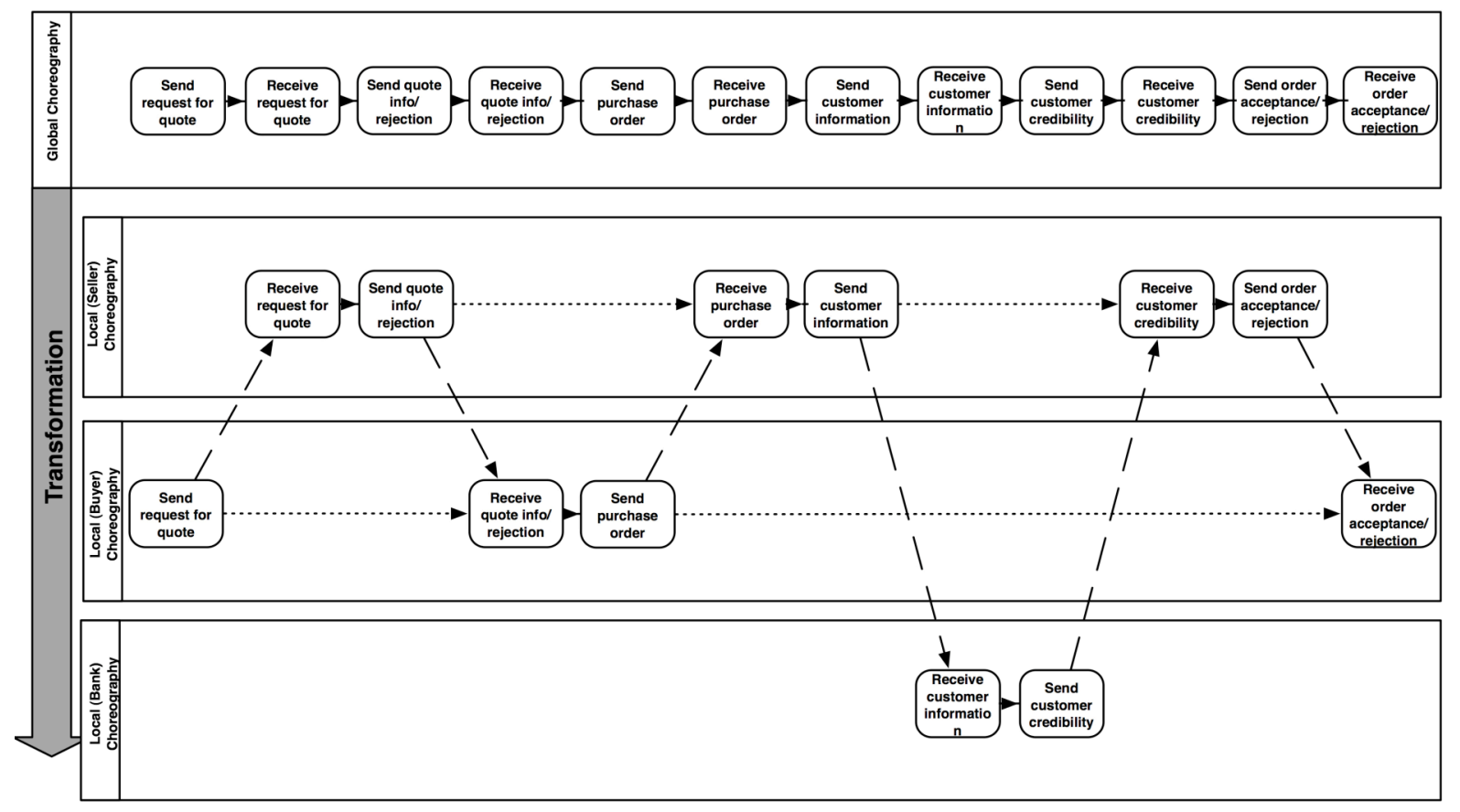

Fig. 5. Choreography model of the example case

Each pool below the global choreography, shown in Fig. 5, comprises the choreography of the single interaction activities of one participating partner. This type of choreography is also called local choreography. The basic idea of transforming a global choreography to local choreographies is to assign the activities modeled in the global choreography to single participating partners. This is achieved by ignoring activities, which are not related to the particular party, and is necessary in order to ensure a decentralized execution of the interorganizational business process steps. Several approaches for modeling global and local choreography have been proposed based on different formalisms. E.g., in the approach of van der Aalst and Weske (van der Aalst and Weske, 2001) the partners first agree on the overall structure of the global choreography. The key tasks are identified as well as control and data dependencies between them. As the second step, the global choreography is partitioned along organizational lines, where the partners are assigned to be responsible for completing parts of the process. The partitioning creates a set of process fragments for each business partner (local choreography).

The orchestration scenario additionally adds to the local choreographies invocations of internal activities, executed either by internal information systems or by human beings. Looking at the example case the following invocations are added e.g., to the local choreography of the seller. After receiving the request for quote the orchestration of the seller invokes the activity to check whether the requested product is listed in his catalog. If so, the orchestration invokes the activity to check whether the product is in stock. In case that the product is out of stock the orchestration invokes the activity of checking if it is possible to produce the product in time. In case that the product is either in stock or may be produced in time, the orchestration invokes the activity of determining the price before sending a quote to the buyer. Additionally, the invocation of the activity to check the credibility of the customer is added to the orchestration before sending an order acceptance or rejection to the buyer. The seller orchestration of the example case is shown in the top pool of Fig. 6, having highlighted the invocation activities of the orchestration. 


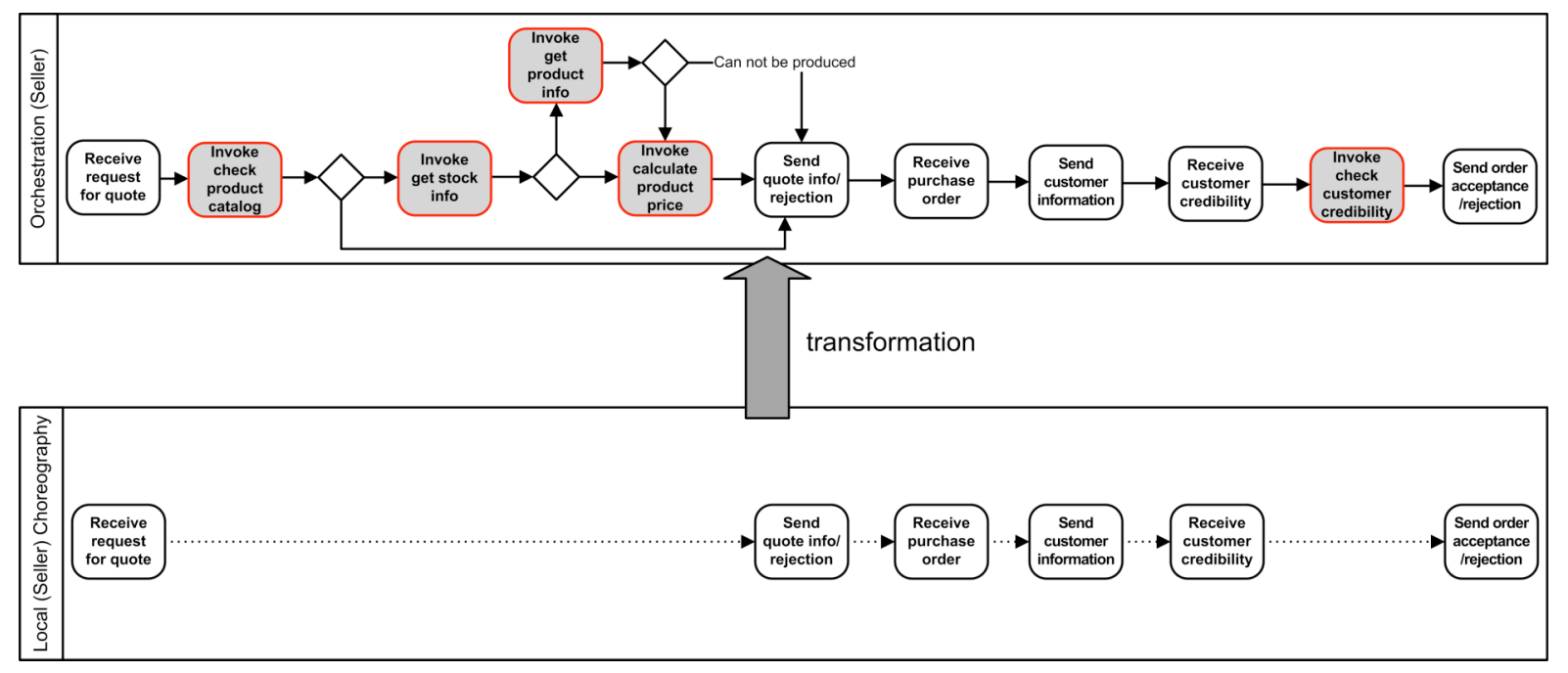

Fig. 6. Transformation of the seller's local choreography to the orchestration

The transformation of local choreography to orchestration involves the extension of the local choreography with data transformation activities and/or invocations of activities executed either by internal information systems or human beings. The extensions of the local choreography must preserve the interaction logic of the global choreography (van der Aalst and Weske, 2001). To guarantee the successful transformation, the notion of inheritance of dynamic behavior appears to be useful (Basten and van der Aalst, 2001). The notion is based on two transformation methods: hiding - where the effects of the tasks internal to the orchestration are ignored - and blocking - where the tasks internal to the orchestration are not executed. Thus, an orchestration inherits from a local choreography if after applying the two transformation methods it is not possible to distinguish between the behaviors of the two processes. In Bussler's approach (Bussler, 2002) the local choreography (public process) is transformed to the orchestration (private process) by using the concept of binding. A binding is a process that connects public processes on the one side and private processes on the other side. Since a binding is a process, its definition contains process steps, which receive and send messages from the public processes to the private process and vice versa. The binding is used to perform message transformations (such as document format transformations), or even to consume or produce messages. In this way, the binding allows the public processes to completely operate on their specific formats, and private processes to completely operate on the normalized format.

Now we have a short look at the inverse transformations. The transformation of orchestration to local choreography assumes the abstraction of activities invoked by the orchestration. The result is a local choreography, specifying the interaction tasks defined in the orchestration. To support the transformation, Basten and van der Aalst (Basten and van der Aalst, 2001) use the previously described methods of hiding and blocking to abstract the internal behavior. If all internal tasks of an orchestration are hidden or blocked, then a local choreography is obtained. Bussler defines the transformation by using the binding process, which receives messages from the private process (orchestration), transforms them and sends them to the public process (local choreography).

The transformation of local choreography to global choreography can be realized by relating activities of the different local choreographies with each other, representing communication channels. These communication channels can be considered to be synchronous, i.e., blocking the further execution, or asynchronous, i.e., non-blocking the further execution. In either case the local choreographies related by communication channels can be transformed into a single workflow model, i.e., the global choreography. An approach based on workflownets has been proposed by van der Aalst (van der Aalst, 2002, van der Aalst, 1999), where in particular the asynchronous communication has been applied. Piccinelli (Piccinelli et al., 
2002) proposes another approach for the transformation, particularly usable for peer-to-peer collaborations. In his approach, the global choreography is obtained dynamically, by reconciling the interaction tasks of the local choreographies in a step-wise manner. The method implies the use of the blocking and hiding techniques. Opposite to van der Aalst's notion of the projection inheritance, where a task is hidden explicitly, Picinnelli explicitly represents the corresponding virtual task of the complementary process.

\section{Executing Inter-Organizational Business Processes}

\section{(Contributing authors: Koschmider, Mendling, Verginadis, Weber)}

Once the business processes are understood and modeled, they need to be implemented. Instead of writing programs specific to each process model, it is preferred to load machinereadable process definitions that allow the monitoring and/or execution of the business processes. Such a process definition is a machine-readable model in a declarative syntax, which describes how to execute the inter-organizational business process. For this purpose a lot of different XML-based notations for specifying orchestration and/or choreography have been developed. Whereas BPMN, UML and EPC are graphical languages used in the analysis and design of business processes, the XML-based orchestration/choreography languages are the abstract descriptions of the final output of the analysis and design process. Both BPMN and UML specifications, as introduced in the previous section, can be readily mapped to executable XML languages for process specifications. It follows, that models described in BPMN, UML, EPC have to be transformed to the XML languages for the purpose of executing the process specifications in a workflow environment.

The Business Process Modeling Language (BPML) (Arkin, 2002) is a block-structured metalanguage, which is based on a logical process model that can fully express concurrent, repeating and dynamic tasks and be directly executed via middleware support. BPML describes choreography from the viewpoint of a single partner (orchestration and local choreography).

The Business Process Execution Language for Web Services (BPEL4WS or BPEL for short) (Andrews et al., 2003) is a sub-set of BPML - merging the concepts of the XLANG and WSFL specifications - and describes choreography from the viewpoint of a single partner (local choreography).

The ebXML Business Process Specification Schema (BPSS) (ebXML, n.d.) provides a standard framework for business process specification. It works with the ebXML Collaboration Protocol Profile (CPP) and Collaboration Protocol Agreement (CPA) specifications to bridge the gap between business process modeling and the configuration of ebXML compliant e-business software. BPSS supports the description of the overall collaboration with a single choreography (global choreography).

The Web Services Choreography Description Language (WS-CDL) (Kavantzas et al., 2004) regards inter-organizational cooperation of Web Services participants by defining their common observable behavior. This specification extends the emerging stack of Web Services standards targeted for integrating applications developed in heterogeneous computation environments. WS-CDL supports the description of the overall collaboration with a single choreography (global choreography).

The Web Service Choreography Interface (WSCI) (Arkin et al., 2002) refers to the flow of messages exchanged by a Web Service participating in choreographed interactions with other services. It enables developers, architects and tools to describe and compose a global view of the dynamics of the message exchange by understanding the interactions with the Web Service.

The XML Process Definition Language (XPDL) (XPDL, n.d.) is used to define the objects and attributes contained within a process definition. One of the most important elements of XPDL is a generic construct that supports vendor specific attributes for use within the common 
representation. The XPDL grammar is directly related to these objects and attributes and supports the workflow definition of each participant's internal processes (orchestration).

Recently, BPEL has become the most prominent XML-based language for Web Service orchestration. We present its main concepts as an example to illustrate which kind of aspects need to be specified to make orchestration work. In a simple case, a BPEL process defines partner links, variables, and activities. Partner links represent message exchange relationships between two parties relating a so-called myRole and a partnerRole endpoint. Partner links are used by basic activities that involve Web Service message exchanges. Variables are used to store process data as well as Web Service messages. Scopes are specific structured activities that can define local variables and handlers within their scope. Handlers specify responses to unexpected behavior like time or message events, faults, or compensation. Nesting of structured activities is used to express control flow in BPEL. There are specific structured activities for loops (while), sequential execution (sequence), conditional branching based on data (switch) or events (pick), and concurrent branches (flow). Additional synchronization constraints in a flow can be defined via links. So-called basic activities specify the actual operations of a BPEL process. There are three activities involving Web Services: invoke for calls to a remote Web Service, receive to wait for the receipt of a Web Service message, and reply for responding to a remote request. All these activities reference a partner link and specify input and/or output variables for messages. The correlation set defines those parts of messages that identify the matching process instance. Furthermore, there are activities to assign data to variables (assign), to wait for a certain event before continuing (wait), for doing nothing (empty), to signal a fault (throw), and to end the process abnormally (terminate).

WS-CDL bases its constructions and channel passing on the pi-calculus and BPEL specification represents a merge of IBM's WSFL and Microsoft's XLANG - where XLANG claimed to be influenced by pi-calculus (Milner, 2005) (e.g. compensation transactions (Bocchi et al., 2003)) and WSFL by Petri nets (Reisig and Rozenberg, 1998). For BPEL there is a complete transformation to Petri nets available in (Hinz et al., 2005). Thus, we would like to give a short description of the formalism and description methods underlying the different XML specifications.

Petri nets are a widely accepted graphical language for the specification, simulation and verification of information system behavior. Formally, a Petri net is a directed bipartite graph with nodes and arcs. In elementary Petri nets (place/transition nets) the flow of tokens, representing anonymous objects, defines the process flow. To model inter-organizational business processes, variants of high-level Petri nets have been proposed such as colored Petri nets (Jensen, 1994), Predicate/Transition nets (Pr/T nets) (Genrich and Lautenbach, 1981) or XML nets (Lenz and Oberweis, 2003), and Workflow nets (van der Aalst, 1997). In contrast to elementary Petri nets, in high-level Petri nets tokens are distinguishable (i.e. they have an identity) and enable to describe objects with individual properties. With XML nets the flow of XML documents and the control flow of the underlying business process can be modeled. When utilizing the Petri net as an underlying description method for XML-based specifications, alternative, concurrent, synchronous or sequential control flow semantics including OR, XOR and AND branches and join rules can be expressed. For Petri nets there is a rich set of analysis techniques available in order to verify soundness and other desirable properties of a process. Woflan is an example of an open source tool that performs such analysis (Verbeek et al., 2001).

The pi-calculus is an process algebra with a number of basic concepts, in terms of which interaction behavior can be rigorously described. A pi-calculus process supports sequential, conditional or concurrent control flow. By sending information to another process the name of the channel for the other process to respond is included in the message. Pi-calculus is qualified as an underlying formalism for XML-based specifications because of its sequential, conditional, parallel, and recursive process behaviors. Further, it supports message-based 
communication. The applicability of Pi-calculus for the formalization of workflow patterns has been studied in (Puhlmann and Weske, 2005).

The mapping from a conceptual inter-organizational process model as introduced in section 4 to an implementation, and therefore to one of the XML specification languages as introduced in this section, involves several issues that need to be considered; these include the mapping of control flow, the generation of an implementation process for each party, and dealing with the problem of different levels of abstraction. The control flow transformation is rather specific for a mapping from a conceptual to an implementation model. As an example, we sketch some of the ideas of the mapping from graph-based BPMN to block-structured BPEL as presented in (White, 2004). A more general discussion of transformations between graph- and block-oriented business process modeling languages can be found in (Mendling et al., 2005). The general idea of the mapping from BPMN to BPEL is to generate a separate BPEL process for each swim lane. Start events of BPMN are basically transformed to reactive basic activities in BPEL such as receive, wait, or pick, while end events map to reply, invoke, throw, or terminate. BPMN tasks are transformed to corresponding BPEL basic activities. The mapping of complex control flow as represented by gateways in BPMN is not trivial. Basically, there are two options: to express all control flow via links, or to identify structured blocks that are mapped to structured activities in BPEL (Mendling et al., 2005). Both come with a specific problem: control flow expressed with links must be acyclic, which is not always granted in a BPMN diagram, and the BPMN model does not always follow a structure that can be mapped to BPEL structured activities. White (White, 2004) proposes to identify the extent of BPEL structured activities by using so-called token analysis. The idea behind that is related to graph reduction rules (Sadiq and Orlowska, 1999): identify a structured block in the BPMN process graph and map it to a BPEL structured activity until no more structured blocks can be identified. Transformation to BPEL links and BPEL structured activities can be combined in order to optimize the resulting BPEL code (Mendling et al., 2005).

\section{Conclusions}

There is a clear trend in business and industry that companies increasingly participate in cooperative networks, for their own benefit. These networks may be temporary, like in civil engineering projects, or more or less permanent, like in supply chains and health care. In both cases, however, they are dynamic: during the existence of the network, members may join and leave. In fact, participation in such networks is becoming the normal way of operation for companies. In order to meet the requirements and challenges of participating in networks, companies must be able to cooperate effectively and efficiently. This ability comprises a number of things, at different levels of operation and management. Many of them have been studied in recent research projects, but also many still wait to be investigated. We have tried, in this paper, to provide an overview of what has been established and what is going on regarding the cooperation of enterprises in networks. Our focus has been the modeling of cooperation, from the business level down to the implementation level. Inevitably, this overview is 'colored' by the preferences of the authors and the many contributors. At the same time, however, we are confident that it is a useful source of knowledge for those who want to have a quick insight in the relevant aspects of cooperation, and in many well known modeling approaches and techniques. Hopefully, it is also an inspiring source for those who want to investigate yet unsolved or unsatisfactorily solved problems. Although developments, both in theory and in practice, will go on, we do not expect landslides. Particularly for practice, the value of this report will therefore last for a considerable time.

\section{References}

ALBANI, A., BAZIJANEC, B., TUROWSKI, K. \& WINNEWISSER, C. (2004a) Component Framework for Strategic Supply Network Development. 8th East-European Conference on Advances in Databases and Information Systems (ADBIS-04), LNCS 3255. Budapest, Hungary, Springer Verlag. 
ALBANI, A., DIETZ, J. L. G. \& ZAHA, J. M. (2005) Identifying Business Components on the basis of an Enterprise Ontology. Interop-Esa 2005 - First International Conference on Interoperability of Enterprise Software and Applications. Geneva, Switzerland.

ALBANI, A. \& MÜSSIGMANN, N. (2005) Evaluation of supply networks. IN MEERSMAN, R., TARI, Z. \& AL., E. (Eds.) International Workshop on Modelling Inter-Organisational Systems (MIOS), in conjunction with the OTM 2005 Federated Conferences, LNCS. Larnaca, Cyprus, Springer.

ALBANI, A., WINNEWISSER, C. \& TUROWSKI, K. (2004b) Dynamic Modelling of Demand Driven Value Networks. On The Move to Meaningful Internet Systems and Ubiquitous Computing 2004: CoopIS, DOA and ODBASE, LNCS. Larnaca, Cyprus, Springer Verlag.

ALONSO, G., CASATI, F., KUNO, H. \& MACHIRAJU, V. (2003) Web Services: Concepts, Architectures, and Applications, Springer Verlag.

ANDERSON, P., GOVINDARAJAN, V., TRIMBLE, C. \& VEERMAN, K. (2002) Cisco Systems (B) Maintaining an Edge in E-Business. Excellence in Strategic Innovation Series, No. 1-0002.

ANDREWS, T., CURBERA, F., DHOLAKIA, H., GOLAND, Y., KLEIN, J., LEYMANN, F., LIU, K., ROLLER, D., SMITH, D., THATTE, S., TRICKOVIC, I. \& WEERAWARANA, S. (2003) Business Process Execution Language for Web Services, Version 1.1., BEA Systems, IBM Corp., Microsoft Corp., SAP AG, Siebel Systems.

ARKIN, A. (2002) Business Process Modeling Language (Version 1.0).

ARKIN, A., ASKARY, S., FORDIN, S., JEKELI, W., KAWAGUCHI, K., ORCHARD, D., POGLIANI, S., RIEMER, K., STRUBLE, S., TAKACSI-NAGY, P., TRICKOVIC, I. \& ZIMEK, S. (2002) Web Service Choreography Interface (WSCI) 1.0.

BARROS, A., DUMAS, M. \& OAKS, P. (2005) Standards for Web Service Choreography and Orchestration: Status and Perspectives. IN BUSSLER, C. \& HALLER, A. (Eds.) Workshop on Web Service Choreography and Orchestration for Business Process Management hold in conjunction with the Business Process Management conference (BPM). Nancy, France, Springer, LNCS.

BASTEN, T. \& VAN DER AALST, W. M. P. (2001) Inheritance of Behavior. Journal of Logic and Algebraic Programming, 47, 47-145.

BOCCHI, L., LANEVE, C. \& ZAVATTARO, G. (2003) A calculus for long-running transactions. Proceedings of 6th Intenational Conference on Formal Methods for OpenObject Based Distributed Systems. Springer, LNCS.

BRUFFEE, K. A. (1995) Cooperative Learning versus Collaborative Learning. Change, 27, 12-18.

BURTLER, P., HALL, T. W., HANNA, A. M., MENDONCA, L., AUGUSTE, B., MANYIKA, J. \& SAHAY, A. (1997) A Revolution in Interaction. The McKinsey Quarterly, 1/97, 4-23.

BUSSLER, C. (2002) The Application of Workflow Technology in Semantic B2B Integration. Distributed and Parallel Databases. Kluwer Academic Publishers.

DAVENPORT, T. H. (1992) Process Innovation: Reengineering Work Through Information Technology. Harvard Business School Press.

DAVENPORT, T. H. (1998) Putting the Enterprise into the Enterprise System. Harvard Business Review, 76, 121-131.

DAVENPORT, T. H. \& SHORT, J. E. (1990) The New Industrial Engineering: Information Technology and Business Process Redesign. Sloan Management Review, 31, 11.

DIJKMAN, R. \& DUMAS, M. (2004) Service-Oriented Design: a Multi-Viewpoint Approach. International Journal of Cooperative Information Systems, 13, 337-368.

DODEL, J. H. (2004) Supply chain integration: Reduction of the logistic criticality in the automotive industry (in German). Unviersity of St. Gallen, Switzerland.

EBXML (n.d.) UN/CEFACT, OASIS. ebXML Business Process Specification Schema version 1.01.

ECL@SS (n.d.) eCl@ss. Version 5.0 ed. 
ETEMAD, H., WRIGHT, R. W. \& DANA, L. P. (2001) Symbiotic International Business Networks: Collaboration between Small and Large Firms. Thunderbird International Business Review, 43, 481-499.

FU, X., BULTAN, T. \& SU, J. (2004) Realizability of Conversation Protocols with Message Contents. Proceedings of IEEE International Conference on Web Services (ICWS).

GENRICH, H. J. \& LAUTENBACH, K. (1981) System modelling with high level petri nets. Theoretical Computer Science, 13, 109-136.

GULATI, R. (1998) Alliances and Networks. Strategic Management Journal, 19, 293-317.

GULATI, R., NOHRIA, N. \& ZAHEER, A. (2000) Strategic networks. Strategic Management Journal, 21, 203-215.

HAGEL III, J. (1996) Spider versus Spider. The McKinsey Quarterly, 1.

HAMMER, M. (1990) Reengineering Work: Don't Automate, Obliterate. Harvard Business Review, 68, 104-112.

HERMAN, J. (2002) Global Value Webs. Supply Chain Management Review, July/August.

HINZ, S., SCHMIDT, K. \& STAHL, C. (2005) Transforming BPEL to Petri Nets. IN VAN DER AALST, W. M. P., BENATALLAH, B., CASATI, F. \& CURBERA, F. (Eds.) Third International Conference on Business Process Management (BPM). Nancy, France, Springer.

HOULIHAN, J. B. (1985) International Supply Chain Management. International Journal of Physical Distribution and Logistics Management, 15, 22-38.

JARILLO, C. J. (1988) On Strategic Networks. Strategic Management Journal, 9, 31-41.

JENSEN, K. (1994) An introduction to the theoretical aspects of coloured petri nets. IN DE BAKKER, J. W., DE ROEVER, W. P. \& ROZENBERG, G. (Eds.) A Decade of Concurrency - Reflections and Perspectives. Springer, LNCS.

JONES, T. \& RILEY, D. (1985) Using Inventory for Competitive Advantage through Supply Chain Management. International Journal of Physical Distribution and Logistics Management, 5, 16-22.

KAPLAN, R. B. \& MURDOCK, L. (1991) Core process redesign. The McKinsey Quarterly, 27-34.

KAVANTZAS, N., BURDETT, D., TITZINGER, G., FLETCHER, T. \& LAFON, Y. (2004) Web Services Choreography Description Language (WS-CDL) 1.0, W3C Working Draft 17 Dec.

KELLER, G., NÜTTGENS, M. \& SCHEER, A.-W. (1992) Semantische Prozeßmodellierung auf der Grundlage "Ereignisgesteuerter Prozeßketten (EPK), Saarbrücken.

KOPANAKI, E., SMITHSON, S., KANELLIS, P. \& MARTAKOS, D. (2000) The Impact of Inter-organizational Information Systems on the Flexibility of Organizations. Proceedings of the Sixth Americas Conference on Information Systems (AMCIS). Long Beach, CA.

KRAEMER, K. L., DEDRICK, J. \& YAMASHIRO, S. (2000) Refining and Extending the Business Model With Information Technology: Dell Computer Corporation. The Information Society, 16, 5-21.

LAMBERT, D. M. \& COOPER, M. C. (2000) Issues in Supply Chain Management. Industrial Marketing Management, 29, 65-83.

LENZ, K. \& OBERWEIS, A. (2003) Interorganizational business process management with XML nets. IN EHRIG, H., REISIG, W., ROZENBERG, G. \& WEBER, H. (Eds.) Petri Net Technology for Communication-Based Systems, Advances inPetri Nets. Springer, LNCS.

MALONE, T. W. \& LAUTBACHER, R. J. (1998) The Dawn of the E-Lance Economy. Harvard Business Review, 145 - 152.

MALONE, T. W., YARES, J. \& BENJAMIN, R. I. (1987) Electronic Markets and Electronic Hierarchies. Communications of the ACM, 30, 484-497.

MENDLING, J., LASSEN, K. \& ZDUN, U. (2005) Transformation Strategies between BlockOriented and Graph-Oriented Process Modelling Languages. Vienna University of Economics and Business Administration. 
MILES, R. E. \& SNOW, C. C. (1984) Fit, Failure and The Hall of Frame. California Management Review, 26, 10-28.

MILNER, R. (2005) A calculus of communicating systems. Springer, LNCS.

MUTH, P., WODTKE, D., WEISSENFELS, J., DITTRICH, A. K. \& WEIKUM, G. (1998) From centralized workflow specification to distributed workflow execution. Journal of Intelligent Information Systems, 10.

MÜSSIGMANN, N. \& ALBANI, A. (2006) Supplier Network Management: Evaluating and Rating of Strategic Supply Networks. 21st ACM Symposium on Applied Computing (SAC 2006). Dijon, France.

OSTERLOH, M. \& FROST, J. (2003) Process management as core competence (in German), Wiesbaden, Gabler Verlag.

PICCINELLI, G., EMMERICH, W., ZIRPINS, C. \& SCHÜTT, K. (2002) Web Service Interfaces for Interorganisational Business Processes - An Infrastructure for Automated Reconciliation. Proceedings of the 6th International Enterprise Distributed Object Computing Conference (EDOC).

PICOT, A., REICHWALD, R. \& WIGAND, R. T. (2003) The boundless Enterprise - information, organization and management (in German), Wiesbaden, Gabler Verlag.

PINE, B. J., VICTOR, B. \& BOYNTON, A. C. (1993) Making Mass Customization Work. Havard Business Review, 36, 108-119.

PRAHALAD, C. K. \& GARY, H. (1990) The Core Competence of the Corporation. Harvard Business Review, 68, 79-91.

PUHLMANN, F. \& WESKE, M. (2005) Using the Pi-Calculus for Formalizing Workflow Patterns. IN VAN DER AALST, W. M. P., BENATALLAH, B., CASATI, F. \& CURBERA, F. (Eds.) Third International Conference on Business Process Management (BPM). Nancy, France, Springer Verlag.

REISIG, W. \& ROZENBERG, G. (1998) Lectures on Petri Nets: Basic Models. 1 ed., Springer, LNCS.

ROSS, J. W. \& VITALE, M. R. (2000) The ERP Revolution: Surviving vs. Thriving. Information Systems Frontiers, 2, 233-241.

SADIQ, W. \& ORLOWSKA, M. E. (1999) Applying Graph Reduction Techniques for Identifying Structural Conflicts in Process Models. IN JARKE, M. \& OBERWEIS, A. (Eds.) 11th Conference on Advanced Information Systems Engineering (CAiSE'99). Heidelberg, Germany, Springer, LNCS.

SCHEER, A.-W. (1998) ARIS - Business Process Frameworks, Berlin, Springer.

SCHEER, A.-W. (2000) ARIS - Business Process Modeling, Berlin, Springer.

SCHEER, A.-W., THOMAS, O. \& ADAM, O. (2005) Process Modeling Using Event-Driven Process Chains. IN DUMAS, M., VAN DER AALST, W. M. P. \& TER HOFSTEDE, A. $\mathrm{H}$. (Eds.) Process Aware Information Systems: Bridging People and Software Through Process Technology. Wiley Publishing.

SCOTT, J. E. \& VESSEY, I. (2000) Implementing Enterprise Resource Planning Systems: The Role of Learning from Failure. Information Systems Frontiers, 2, 213-232.

TAPSCOTT, D., TICOLL, D. \& LOWY, A. (2000) Digital Capital: Harnessing the Power of Business Webs, Boston.

THORELLI, H. B. (1986) Networks: Between Markets and Hierarchies. Strategic Management Journal, 7, 37-51.

UML (n.d.) OMG Unified Modelling Language, Version 2.0.

UN/CEFACT (n.d.) UN/CEFACT TMG: Modeling Methodology-Metamodel.

UN/SPSC (n.d.) UN/SPSC.

VAN DER AALST, W. M. P. (1997) Verification of Workflow Nets. IN AZÉMA, P. \& BALBO, G. (Eds.) Applications and Theory of Petri Nets. Springer Verlag.

VAN DER AALST, W. M. P. (1999) Interorganizational Workflows: An Approach based on Message Sequence Charts and Petri Nets. Journal of Systems Analysis - Modeling Simulation, 34, 335-367. 
VAN DER AALST, W. M. P. (2002) Inheritance of Interorganizational Workflows to Enable Business-to-Business E-commerce. Journal of Electronic Commerce Research, 2, 195-231.

VAN DER AALST, W. M. P., TER HOFSTEDE, A. H., KIEPUSZEWSKI, B. \& BARROS, A. (2003) Workflow Patterns. Distributed and Parallel Databases, 14, 5-51.

VAN DER AALST, W. M. P. \& WESKE, M. (2001) The P2P approach to Interorganizational Workflows. IN K.R.DITTRICH, A.GEPPERT \& M.C.NORRIE (Eds.) Conference on Advanced Information Systems Engineering (CAiSE). Interlaken, Lecture Notes in Computer Science (LNCS).

VERBEEK, H. M. W., BASTEN, T. \& VAN DER AALST, W. M. P. (2001) Diagnosing Workflow Processes using Woflan. The Computer Journal, 44, 246-279.

WHITE, S. (2004) Business Process Modeling Notation. The Business Management Initiative.

WILLIAMSON, O. E. (1991) Comparative Economic Organization: The Analysis of Discrete Structural Alternatives. Adminstrative Science Quarterly, 36, 269-296.

WILSON, T. (2001) Supply Chain Debacle. Internetweek, 1-2.

XPDL (n.d.) WfMC. Workflow Process Definition Interface - XML Process Defintion Language. WFMC-TC-1025.

YI, X. \& KOCHUT, K. J. (2004) Process Composition of Web Services with Complex Conversation Protocols: a Colored Petri Nets Based Approach. Proceedings of the Design, Analysis, and Simulation of Distributed Systems Symposium at Advanced Simulation Technology Conference. 